\title{
Improving the Pediatric to Adult Care Transition Experience: Recommendations From Young Adults With Complex Care Needs: A Scoping Review Protocol
}

\author{
Monique Cassidy1, MEd; Shelley Doucet2, 3, PhD, RN; Alison Luke², 3, PhD; and \\ Alex Goudreau ${ }^{3,4}$, MA, MLIS \\ ${ }^{1}$ School of Graduate Studies, Interdisciplinary Studies, University of New Brunswick \\ ${ }^{2}$ Department of Nursing and Health Sciences, University of New Brunswick \\ 3 University of New Brunswick Saint John Collaboration for Evidence-Informed Healthcare: A \\ Joanna Briggs Institute Centre of Excellence \\ ${ }^{4}$ University of New Brunswick Libraries \\ https://doi.org/10.15273/hpj.v1i1.10643
}

\begin{abstract}
With advancements in modern medicine, an increasing number of youth with complex care needs (CCN) survive into adulthood. As service demands increase for this group, challenges exist on how to best facilitate meeting their needs as they transition from pediatric to adult health care. There is growing evidence of young adults describing their transition experiences and suggesting improvements to the design and delivery of these services. By synthesizing the existing literature, an increased understanding can be gained about the recommendations of those who have recently transitioned from pediatric to adult health care, thus improving both patient outcomes and experiences. This scoping review aims to comprehensively map recommendations on how to improve the transition from pediatric to adult health care based on the experiences of young adults (aged 18-30) with CCN. This study protocol outlines a scoping review of peer-reviewed and grey literature, following the Joanna Briggs Institute (JBI) scoping review methodology. Literature will be identified using a comprehensive search strategy developed by a JBI-trained librarian. Papers involving primary studies with recommendations of young adults recently transitioned from pediatric to adult care will be included. Search strategy results will be screened by two independent reviewers and included studies will have duplicates removed and charted according to a modified PRISMA flow diagram. Working with the Centre for Research in Integrated Care at the University of New Brunswick, knowledge translation activities will involve targeted communication channels to a variety of knowledge users, such as researchers, clinicians, and policymakers.
\end{abstract}

Keywords: pediatric to adult transition, transition experience, complex care needs, scoping review, quality improvement, patient engagement 


\section{Improving the Pediatric to Adult Care Transition Experience: Recommendations From Young Adults With Complex Care Needs: A Scoping Review Protocol}

Young adulthood (YA) is a complex time when life transition dilemmas arise concerning education, employment, health engagement, and relationships (Al-Yateem, 2012; Cheak-Zamora \& Teti, 2015; Vincent, 2017). It is an important life development stage since it "determines the young adult's position within society" (de JongGierveld, 2001, p. 98). Due in part to modern health care advances, a high number of young adults are living with chronic conditions in North America (Cohen et al., 2011; Kaufman et al., 2007; Pinzon et al., 2006). Youth with medical complexities are also more likely to survive into adulthood (Cohen et al., 2011; Kaufman et al., 2007; Wise, 2004). As a result, Chamberlain and Kent (2005) reinforce the urgent need to study and implement effective services for youth transitioning from pediatric to adult care to set them on a path for optimal life outcomes. When health care transition interventions are delivered in a structured and informed way, improvements in patient experience, health care outcomes, and population health can result (Schmidt et al., 2020). A synthesis of research is required to understand the transition process from pediatric to adult care from the perspective of young adults with medical complexities, specifically their recommendations on how to improve transition policies and practices. While youth from this population represent only a fraction of those transitioning from pediatric to adult care, they encounter more challenges with the process, and thus their recommendations may contribute to transition process improvements for other populations (White \& Cooley, 2018).

To date, reviews on the transition from pediatric to adult services have largely reported on the experiences of youth through the lens of caregivers and care providers, ignoring the voices of the young adults who have experienced transition in care (Betz et al., 2013; Okumura et al., 2015).
The transition from pediatric to adult care is categorized as the purposeful and planned process that involves the movement from child-centred to adult-oriented care systems (Blum, 2002). This transition process can be challenging for youth with complex care needs (CCN). CCN are defined as "multidimensional health and social care needs in the presence of a recognized medical condition or where there is no unifying diagnosis. CCN are individual and contextualized, are continuing and dynamic, and are present across a range of settings, impacted by healthcare structure" (Brenner et al., 2018, p. 1647). As a result of these complexities, youth with chronic health conditions are significant users of health care resources (Pinzon et al., 2006). Examples of conditions associated with CCN include diabetes, cerebral palsy, epilepsy, spina bifida, and mental health conditions (e.g., depression, anxiety, mood disorders; Brenner et al., 2018; New Brunswick Health Council [NBHC], 2017). In North America, approximately $15-18 \%$ of youth have at least one chronic condition (Arim et al., 2015; Kaufman et al., 2007).

Evidence suggests that improving the transition experience of young adults with CCN is challenging, since this population often faces service provision gaps and barriers to accessing care (Nelson, 2016; Rich, 2017). Understanding the health care experiences of this population is a crucial first step in driving health care improvement (Luxford \& Sutton, 2014). White and Cooley (2018) suggest more research is needed regarding health care transitions with a focus on experiences of youth, young adults, and families, as well as examining transition outcomes from a population health perspective. Currently, very little research focuses on the perspectives of young adults with various CCN when making recommendations for improvement in the transition process. To our knowledge, there is no comprehensive review focused uniquely on capturing improvement recommendations of $\mathrm{CCN}$ patients from the lens of young adults who have recently transitioned from pediatric to adult health care. 
Given the growing body of literature on YA transition, a number of systematic reviews have been conducted in the following transition areas:

- health care for youth with special health care needs (Bloom et al., 2012),

- care of children and youth with type 1 diabetes (Nakhla \& Daneman, 2012),

- young peoples' experiences of care from child to adult mental health services (Nelson, 2016; Rich, 2017),

- evaluation of research on adolescents and emerging adults with special health care needs perspectives (Betz et al., 2013),

- continuity of care applications to transition research and clinical practice using the International Classification of Functioning, Disability and Health (ICF) framework for youth with chronic health conditions (Nguyen et al., 2018), and

- meta-synthesis of experiences when transferring from pediatric to adult wards within a hospital setting (Fegran et al., 2014).

The reviews published to date have limited areas of scope, such as focusing only on the outcomes of transition (e.g., adherence; Nakhla \& Daneman, 2012), concentrating on transition models or systems issues (Amaria et al., 2011; Nelson, 2016), exclusively focusing on Organisation for Economic Co-operation and Development (OECD) free economic market country settings (Rich, 2017), or being restricted to one patient population or sector (e.g., autism patients in the context of higher education; Vincent, 2017). There is also insufficient inclusion of youths' voices compared to other perspectives such as that of caregivers (Cheak-Zamora \& Teti, 2015; Cohen et al., 2018), with the exception of a review by Betz et al. (2013), which includes the perspectives of adolescents and young adults. This review differs from Betz et al. by including current studies that exclusively feature perspectives of young adults who have already experienced the transition process to adult services, rather than significant adolescent representation. Betz et al.'s (2013) study evaluates research on health care transition for youth with special health care needs. The current scoping review will expand on previous reviews and provide an overview of recommendations on the transition process from the perspective of young adults across geographic locations and study types.

The primary objective of this review is to comprehensively map recommendations on how to improve the transition from pediatric to adult health care based on the experiences of young adults (aged 18-30). It is important to study this period of changeover, given that youth experience disjointed and difficult shifts from pediatric to adult care (Hopper et al., 2014). If left unaddressed, this problem will grow rapidly as the number of young adults with CCN continues to expand (Cohen et al., 2018). Results of this scoping review will address a literature gap and inform future research by producing a clear portrait of synthesized evidence related to improvement recommendations for young adults with CCN progressing from pediatric to adult care.

\section{Methods}

The proposed scoping review will be conducted in accordance with the JBI methodology for scoping review (Peters et al., 2020), as it is recommended to use standardized guidelines in the design and reporting of scoping reviews to ensure reproducibility and robustness. A preliminary search of CINAHL, PubMed, and the JBI Database of Systematic Reviews and Implementation Reports was conducted, and no current or ongoing reviews with the same objective on this topic were identified. In brief, the steps within the JBI scoping review framework are the following: (a) defining and aligning the objective and question/s; (b) developing and aligning the inclusion criteria with the objective/s and question/s; (c) describing the planned approach to evidence searching, selection, extraction, and charting; (d) searching for the evidence; (e) selecting the evidence; (f) extracting the evidence; (g) analyzing the evidence; (h) presenting the results; and (i) summarizing the evidence in relation to the purpose of the review, making conclusions, and noting findings 
(Peters et al., 2020). The following sections describe in detail how the review will adhere to JBI's approach.

\section{Step One: Defining the Review Question}

To align with the study objective, the scoping review will address the following question:

What are the recommendations of young adults (aged 18-30) who recently transitioned from pediatric to adult health care on how to improve the transition experience?

\section{Step Two: Developing the Inclusion Criteria}

The inclusion criteria will consider population, concept, context, and types of evidence sources (Peters et al., 2020).

\section{Population}

The focus of our scoping review is on the experiences of young adults with CCN who have transitioned from pediatric to adult care. YA will be defined as individuals aged 18-30 years. We will accept various terms used in the literature regarding young adults (e.g., emerging adult, adolescent, young man, or young woman). Studies will be excluded if their descriptions include experiences of young adults under the age of 18 or above the age of 30 , given that many young adults enter adult services by age 18 , and transition programs worldwide are completed by the age of 25 (Fegran et al., 2014; Got Transition, n.d.; Kaufman et al., 2007; Nakhla \& Daneman, 2012; Rich, 2017). The decision to cap the population at age 30 will prevent participant recall bias by avoiding lengthy time since their transfer to adult care. The review is not specific to any CCN condition, sex, ethnicity, or other demographic variable.

\section{Concept}

The main study concept is transition experience recommendations from pediatric to adult care. Transition to adulthood from the pediatric system is defined as the "purposeful and planned movement of young adults with chronic physical and medical conditions" (Blum et al., 1993, p. 570), and encompasses emotional, mental, and behavioural health.
Recommendations based on these types of transition experiences must be present in the study to be included and be from the perspective of young adults themselves; studies discussing only experiences without any recommendations will be excluded.

\section{Context}

This review will consider transition experience suggestions of young adults with CCN in all settings, such as hospital, clinic, or community-based settings, as well as across all health sectors. Studies will be excluded if they focus solely on intra-institutional transfer (e.g., intra-hospital). There will not be any additional exclusion limitations placed on this review, as the intent is to explore pediatric to adult health care transition experiences.

\section{Types of Sources}

This scoping review will consider all primary research studies that use a qualitative design. Mixed methods design will only be considered if a qualitative design is included. Systematic reviews that meet the other inclusion criteria will be excluded from the review; however, the references will be handsearched for relevant articles. Grey literature, such as unpublished primary studies and/or evaluation reports, will also be considered for inclusion in this scoping review. Studies published in English, and from around the world, will be considered for inclusion.

\section{Step Three: Developing the Search Approach}

The search strategy follows a multiphase approach and will aim to locate both published and unpublished primary studies. During the first phase, an initial limited search was conducted in MEDLINE (Ovid) and CINAHL (EBSCO) to identify text words in the titles and abstracts as well as index terms from relevant articles. During this process, a librarian (AG) developed a full search strategy for MEDLINE by testing search terms, and only those that yielded unique results were included. The search strategy was externally peer-reviewed by a second librarian (AM) using the Peer Review of Electronic Search Strategies (PRESS) guidelines 
(McGowan et al., 2016). Searches will be conducted using a combination of database index terms and text words, limited by title/abstract. English language limits will be applied; publication date will not be limited. A full search strategy for MEDLINE (Ovid) is provided (see Appendix A). A final search strategy will be adopted for each database.

\section{Step Four: Searching for Evidence}

The databases to be searched include MEDLINE (Ovid), CINAHL (EBSCO), PsycInfo (EBSCO), and Embase (Elsevier). A process to locate sources of unpublished studies and grey literature will be developed using best practices (Bonato, 2018). This will include searching the first 10 pages of Google and Google Scholar, and using relevant keywords to search Dissertations and Theses (ProQuest), institutional repositories, and relevant conference proceedings.

\section{Step Five: Selecting the Evidence}

Following the search, all identified citations will be collated and uploaded into the EndNote citation manager with duplicates removed. Titles and abstracts will then be screened by two independent reviewers for assessment against the inclusion criteria using Covidence review management software. Next, the full text of selected citations will be assessed in detail against the inclusion criteria by two independent reviewers. Reasons for exclusion of full-text studies that do not meet the inclusion criteria will be recorded and reported in the scoping review. Any disagreements that arise between the reviewers at each stage of the study selection process will be resolved through discussion between the two reviewers, or with a third reviewer when needed. Relevant reviews will be tagged within Covidence to search their reference lists. The reference lists of all included studies will be hand-searched for additional studies. The results of the search will be reported in full in the final report and presented in a modified Preferred Reporting Items for Systemic Reviews and Meta-analyses (PRISMA) flow diagram (Tricco et al., 2018). Any deviations from the study protocol will be tracked and reported in the final manuscript.

\section{Step Six: Extracting the Evidence}

Data will be extracted from included papers by the two independent reviewers using a Microsoft Word data extraction tool developed by the reviewers. Extracted data will include specific details about the population, concept, context, study methods, and key findings relevant to the scoping review objective. A draft of the extraction table is provided (see Appendix B). This will be modified and revised as necessary during the extraction of included studies. Modifications will be detailed in the full scoping review report. Any disagreements that arise between the two reviewers will be resolved through discussion, or with a third reviewer as needed. Authors of research articles and other sources of literature may be contacted to request additional or missing data where needed.

\section{Step Seven: Synthesizing the Evidence}

Data extracted from included papers will be presented in a tabular form (Peters et al., 2020). The table will feature results (e.g., specifying participant subgroup conditions and improvement recommendations, year of publication, research methods, and study purpose). Data will be synthesized based on types of recommendations (e.g., health service need) which will then be pulled together into corresponding themes using qualitative content analysis. Results will be categorized under recommendation types (e.g., service delivery program) accompanied by a clear explanation of classification criteria.

\section{Step Eight: Presenting the Results}

The data extracted from relevant published and unpublished literature will be presented in a tabular form that is aligned with the objective of this scoping review (e.g., recommendations). Data displayed will include the author and year of publication, country of origin, type of literature, research design, methods, population(s), sample size, setting, sector, and transition recommendation(s). 


\section{Step Nine: Summarizing the Evidence}

A narrative summary will accompany the tabulated data and will describe how the results relate to the review objective and question.

\section{Patients and Public Involvement Statement}

Patient opinions from a local advisory group will be consulted to design a collaborative strategy for disseminating finding results.

\section{Results/Discussion and Dissemination}

This scoping review aims to expand transition improvement research by synthesizing recommendation evidence from young adults with CCN as experienced by those already transitioned. Further insights into patient suggestions on improving their care will be uniquely captured in this process. To our awareness, this is the first scoping review to focus on capturing the improvement recommendations of patients who have CCN from the perspective of young adults who have recently transitioned from pediatric to adult health care. A limitation of this review is that it will not assess studies for quality; scoping reviews do not involve assessing included studies for measures of rigour (Peters et al., 2015). Also, limitations may exist regarding the number of databases searched and will be addressed by explicitly acknowledging that searching other databases may have identified additional relevant studies. Searching four subject databases will help address selection bias, and database selection was guided by the topic. A search of the grey literature will also be conducted to identify additional articles. The search strategy consists of terms appropriately describing the research question, and was peerreviewed by another JBI-trained librarian. Due to limited resources for translation, articles published in languages other than English will be excluded.

Furthermore, by not having inclusion or exclusion criteria restrictions on specific young adult conditions, sex, ethnicity, or other demographic variables, study results will be of interest to readers from various health backgrounds and disciplines.

\section{Recommendations}

Recommendations from young adults can guide knowledge users, such as researchers, clinicians, and policy-makers, toward overcoming health transition service inequalities. Synthesizing recommendations from youth with a variety of chronic conditions has proven a useful tool for policy-makers when addressing health consequences for large populations (Arim et al., 2015). A recent systematic review by Barnert et al. (2019) reinforces the pressing need to develop population health outcome measures (e.g., improving health access) for youth with medical complexities. To fulfill this research need, implications from this review will produce specific ways to improve population health service delivery and indicate key areas for future research directions. Policy-makers from all health sectors involved with transitions from pediatric to adult health care will be encouraged to incorporate results from this review into their quality improvement planning and policy initiatives. Review findings will be disseminated through conference proceedings and a peerreviewed publication.

\section{Author Note}

All authors (MC, SD, AL, and AG) provided critical revisions to the work. This included reading and approving the final manuscript. The views expressed in this study reflect the authors' own perspective. The authors declare no conflict of interest. Not commissioned; externally peer reviewed. We would like to thank Alison Manley, Health Sciences Librarian with Horizon Health Network, for peer-reviewing the search strategy for this scoping review. 


\section{References}

Al-Yateem, N. (2012). Child to adult:

Transitional care for young adults with cystic fibrosis. The British Journal of Nursing, 21(14), 850-854. https://doi.org/10.12968/bjon.2012.21 .14 .850

Amaria, K., Stinson, J., Cullen-Dean, G., Sappleton, K., \& Kaufman, M. (2011). Tools for addressing systems issues in transition. Healthcare Quarterly, 14(Spec No. 3), 72-76.

Arim, R. G., Kohen, D. E., Brehaut, J. C., Guèvremont, A., Garner, R. E., Miller, A. R., McGrail, K., Brownell, M., Lach, L. M., \& Rosenbaum, P. L. (2015). Developing a non-categorical measure of child health using administrative data. Statistics Canada. https://www150.statcan.gc.ca/n1/pub /82-003-x/2015002/article/14140eng.htm

Barnert, E. S., Coller, R. J., Nelson, B. B., Thompson, L. R., Tran, J., Chan, V., Padilla, C., Klitzner, T.S., Szilagyi, M., \& Chung, P. J. (2019). Key population health outcomes for children with medical complexity: A systematic review. Maternal and Child Health Journal, 23(9), 1167-1176. https://doi.org/10.1007/s10995-01902752-1

Betz, C. L., Lobo, M. L., Nehring, W. M., \& Bui, K. (2013). Voices not heard: A systematic review of adolescents' and emerging adults' perspectives of health care transition. Nursing Outlook, 61(5), 311336.

https://doi.org/10.1016/j.outlook.2013 .01 .008

Bloom, S. R., Kuhlthau, K., Van Cleave, J., Knapp, A. A., Newacheck, P., \& Perrin, J. M. (2012). Health care transition for youth with special health care needs. Journal of Adolescent Health, 51(3), 213-219. https://doi.org/10.1016/j.jadohealth.2 012.01.007
Blum, R. W. (2002). Introduction. Improving transition for adolescents with special health care needs from pediatric to adult-centered health care. Pediatrics, 110, 1301-1303.

Blum, R. W., Garell, D., Hodgman, C. H., Jorissen, T. W., Okinow, N. A., Orr, D. P., \& Slap, G. B. (1993). Transition from childcentered to adult health-care systems for adolescents with chronic conditions: A position paper of the Society for Adolescent Medicine. Journal of Adolescent Health, 14(7), 570-576.

Bonato, S. (2018). Searching the grey literature: A handbook for searching reports, working papers, and other unpublished research. Rowman \& Littlefield.

Brenner, M., Kidston, C., Hilliard, C., Coyne, I., Eustace-Cook, J., Doyle, C., Begley, T., \& Barrett, M. J. (2018). Children's complex care needs: A systematic concept analysis of multidisciplinary language. European Journal of Pediatrics, 177(11), 1641-1652. https://doi.org/10.1007/s00431-0183216-9

Chamberlain, M. A., \& Kent, R. M. (2005). The needs of young people with disabilities in transition from paediatric to adult services. Europa Medicophysica, 41(2), 111-123.

Cheak-Zamora, N. C., \& Teti, M. (2015). "You think it's hard now ... It gets much harder for our children": Youth with autism and their caregiver's perspectives of health care transition services. Autism, 19(8), 992-1001. https://doi.org/10.1177/13623613145 58279

Cohen, E., Berry, J. G., Sanders, L., Schor, E. L., \& Wise, P. H. (2018). Status complexicus? The emergence of pediatric complex care. Pediatrics, 141(Supplement 3), S202-S211. https://doi.org/10.1542/peds.2017$1284 \mathrm{E}$ 
Cohen, E., Kuo, D. Z., Agrawal, R., Berry, J. G., Bhagat, S. K. M., Simon, T. D., \& Srivastava, R. (2011). Children with medical complexity: An emerging population for clinical and research initiatives. Pediatrics, 127(3), 529-538. https://doi.org/10.1542/peds.20100910

de Jong-Gierveld, J. (2001). Adolescent behavior: Demographic. In N. J. Smelser, \& P. B. Baltes (Eds.), International encyclopedia of the social and behavioral sciences, (pp. 98-101). Elsevier.

Fegran, L., Hall, E. O. C., Uhrenfeldt, L., Aagaard, H., \& Ludvigsen, M.S. (2014).

Adolescents' and young adults' transition experiences when transferring from pediatric to adult care: A qualitative metasynthesis. International Journal of Nursing Studies, 51(1), 123-135. https://doi.org/10.1016/j.ijnurstu.201 3.02.001

Got Transition. (n.d.). A family toolkit: Pediatricto-adult health care transition. https://www.gottransition.org/resourc e/?hct-family-toolkit

Hopper, A., Dokken, D., \& Ahmann, E. (2014). Transitioning from pediatric to adult health care: The experience of patients and families. Pediatric Nursing, 40(5), 249-252.

Kaufman, M., Pinzon, J., Canadian Paediatric Society, \&Adolescent Health Committee (2007). Transition to adult care for youth with special health care needs. Paediatrics and Child Health, 12(9), 785-788. https://doi.org/10.1093/pch/12.9.785

Luxford, K., \& Sutton, S. (2014). How does patient experience fit into the overall healthcare picture? Patient Experience Journal, 1(1), 20-27.

McGowan, J., Sampson, M., Salzwedel, D. M., Cogo, E., Foerster, V., \& Lefebvre, C. (2016). PRESS peer review of electronic search strategies: 2015 guideline statement. Journal of Clinical Epidemiology, 75, 40-46. https://doi.org/10.1016/j.jclinepi.2016 .01 .021

Nakhla, M. M., \& Daneman, D. (2012). Transitions in the care of children and youth with type 1 diabetes. Diabetes Management, 2(6), 537-547.

Nelson, A. (2016). Paving the way for smooth transitions: Continuity of care from child to adult mental health systems [Unpublished master's thesis]. Simon Fraser University. https://summit.sfu.ca/item/16351

New Brunswick Health Council. (2017). Primary health survey 2017. https://nbhc.ca/sites/default/files/ass ets/images/results_nbhc_2017_primary _health_survey.xlsx

Nguyen, T., Stewart, D., Rosenbaum, P., Baptiste, S., Kraus de Camargo, O., \& Gorter, J. W. (2018). Using the ICF in transition research and practice? Lessons from a scoping review. Research in Developmental Disabilities, 72, 225-239. https://doi.org/10.1016/j.ridd.2017.11 .003

Okumura, M. J., Saunders, M., \& Rehm, R. S. (2015). The role of health advocacy in transitions from pediatric to adult care for children with special health care needs: Bridging families, provider and community services. Journal of Pediatric Nursing, 30(5), 714-723. https://doi.org/10.1016/j.pedn.2015.0 5.015

Peters, M. D. J., Godfrey, C., McInerney, P., Munn, Z., Tricco, A. C., \& Khalil, H. (2020). Chapter 11: Scoping reviews. In E. Aromataris, Z. Munn (Eds.), JBI Manual for Evidence Synthesis. JBI. https://doi.org/10.46658/JBIMES-2012

Peters, M. D. J., Godfrey, C. M., Khalil, H., McInerney, P., Parker, D., \& Soares, C. B. (2015). Guidance for conducting systematic scoping reviews. JBI Evidence Implementation, 13(3), 141146. https://doi.org/10.1097/XEB.0000000 000000050 
Pinzon, J., Harvey, J., Canadian Paediatric Society, \& Adolescent Health Committee (2006). Care of adolescents with chronic conditions. Paediatrics and Child Health, 11(1), 43-48. https://doi.org/10.1093/pch/11.1.43

Rich, E. F. (2017). Getting ready to move on: Considering attachment within young peoples' experiences of preparing to transition out of CAMHS (Publication No. 10765524) [Doctoral dissertation, University of Leicester]. ProQuest Dissertations \& Theses.

Saha, S., Loehrer, S., Cleary-Fisherman, M., Johnson, K., Chenard, R., Gunderson, G., Goldberg. R., Little, J., Resnick, J., Cutts, T., \& Barnett, K. (2017). Pathways to population health: An invitation to health care change agents. 100 Million Healthier Lives. http://www.ihi.org/Topics/PopulationHealth/Documents/PathwaystoPopulat ionHealth_Framework.pdf

Schmidt, A., Ilango, S. M., McManus, M. A., Rogers, K. K., \& White, P. H. (2020). Outcomes of pediatric to adult health care transition interventions: An updated systematic review. Journal of Pediatric Nursing, 51, 92-107. https://doi.org/10.1016/j.pedn.2020.0 1.002

Tricco, A. C., Lillie, E., Zarin, W., O'Brien, K. K., Colquhoun, H., Levac, D., Moher, D., Peters, M. D. J., Horsley, T., Weeks, L., Hempel, S., Akl, E. A., Chang, C., McGowan, J., Stewart, L., Hartling, L., Aldcroft, A., Wilson, M. G., Garritty, C., ... Straus, S. E. (2018). PRISMA extension for scoping reviews (PRISMA-ScR): Checklist and explanation. Annals of Internal Medicine, 169(7), 467473. https://doi.org/10.7326/M180850

Vincent, J. (2017). Being and becoming: Transition from higher education for emerging adults on the autism spectrum [Doctoral thesis]. Lancaster University. https://doi.org/10.17635/lancaster/th esis/76

White, P. H., Cooley, W. C., Transitions Clinical Report Authoring Group, American Academy of Pediatrics, American Academy of Family Physicians, \& American College of Physicians. (2018). Supporting the health care transition from adolescence to adulthood in the medical home. Pediatrics, 142(5), Article e20182587.

https://doi.org/10.1542/peds.20182587

Wise, P. H. (2004). The transformation of child health in the United States. Health Affairs, 23(5), 9-25. https://doi.org/10.1377/hlthaff.23.5.9 


\section{Appendix A}

Search Strategy: Ovid MEDLINE(R) and Epub Ahead of Print, In-Process \& Other Non-Indexed Citations, Daily and Versions(R) 1946 to September 21, 2020

\begin{tabular}{|l|l|l|}
\hline$\#$ & Query & Results \\
\hline 1 & exp Chronic Disease/ & 264078 \\
\hline 2 & Disabled Children/ & 6329 \\
\hline 3 & Health Services for Persons with Disabilities/ & 109 \\
\hline 4 & (chronic adj1 (illness or condition* or disease*).ab,ti. & 94483 \\
\hline 5 & "multimorbid*".ab,ti. & 4800 \\
\hline 6 & (complex* adj3 (care or need* or illness or disease or medical* or condition or & 47451 \\
\hline 7 & health)).ab,ti. & \\
\hline 8 & "medically fragile".ab,ti. & 234 \\
\hline 9 & "Special health* needs ".ab,ti. & 317 \\
\hline 10 & exp "Continuity of Patient Care"/ & 388505 \\
\hline 11 & "transition*".ab,ti. & 246613 \\
\hline 12 & "continuity of care".ab,ti. & 415396 \\
\hline 13 & "continuum of care".ab,ti. & 6856 \\
\hline 14 & 10 OR 11 OR 12 OR 13 & 2545 \\
\hline 15 & Young Adult/ & 659764 \\
\hline 16 & Adolescent/ & 858967 \\
\hline 17 & "young adult* ".ab,ti. & 2036132 \\
\hline 18 & "Adolescen*".ab,ti. & 96111 \\
\hline 19 & "youth*".ab,ti. & 281526 \\
\hline 20 & "juvenile*".ab,ti. & 75624 \\
\hline 21 & 15 OR 16 OR 17 OR 18 OR 19 OR 20 & 82083 \\
\hline 22 & 9 AND 14 AND 21 & 2624021 \\
\hline 23 & limit 22 to english language & 3458 \\
\hline
\end{tabular}




\section{Appendix B}

Data Extraction Template

\begin{tabular}{|l|l|}
\hline Author(s) & \\
\hline Year of Publication & \\
\hline Country of Origin & \\
\hline Type of Literature & \\
\hline Research Design & \\
\hline Study Aim or Purpose & \\
\hline Population(s) & \\
\hline Sample Size & \\
\hline Transition Description & \\
\hline Transition Recommendation(s) & \\
\hline
\end{tabular}

